\title{
Multi-directional colour edge detector using LQS convolution
}

ISSN 1751-9659

Received on 22nd August 2017 Revised 10th January 2018 Accepted on 3rd February 2018 doi: 10.1049/iet-ipr.2017.0921 www.ietdl.org

\author{
Shagufta Yasmin ${ }^{1}$, Stephen J. Sangwine ${ }^{1}$ \\ ${ }^{1}$ School of Computer Science and Electronic Engineering, University of Essex, Wivenhoe Park, Colchester CO4 3SQ, UK \\ 凶E-mail: syasmi@essex.ac.uk
}

\begin{abstract}
A new linear colour image filter based on linear quaternion systems (LQSs) is introduced. It detects horizontal, vertical, left- and right-diagonal edges with a single LQS convolution mask. The proposed filter is a canonic minimal filter of four LQS filters, each with different angles of rotation combined parallel wise. Different angles of rotation are a key features of the new filter such that horizontal, vertical, left, and right-diagonal LQS filter masks rotate pixels through angles $\pi / 2,5 \pi / 2,3 \pi / 2$, and $7 \pi / 2$, respectively. Although, the four LQS masks are combined parallel to make a single LQS mask but derived using four quaternion convolutions, one for each direction of edges, the LQS filter produces a result without the combination of results from four separate edge detectors. This methodology could be generalised to design more elaborate LQS filters to perform other geometric operations on colour image pixels. The proposed filter translates smoothly changing colours to different shades of grey and produces coloured edges in multiple directions, where there is a sudden change of colour in the original image. Another key idea of the proposed filter is that it is linear because it operates in homogeneous coordinates.
\end{abstract}

\section{Introduction}

Colour edge detection has been an active research area for more than three decades. We present a holistic quaternion-based colour edge detector, capable of detecting multi-directional coloured edges with a single linear quaternion (LQ) convolution mask. The proposed filter can be employed on colour images and it is the first example of a filter based on LQ system (LQS) convolution.

Convolution is a fundamental operation in image processing and it is often used as an initial stage of automated image interpretation. Sometimes, strong edges between colours become very faint in luminance and they are hard to detect. The main advantage of colour edge detection is that the edges existing at boundaries between regions of different colours if there is no change in intensity, can be detected while it is not possible in greylevel image processing. Linear grey-scale image convolution filters only scale the pixel values, while most colour image convolution filters process each colour channel separately, they apply scalar convolution and produce the final result by combining the processed channels conventionally. This restricts the convolution operator to the scaling of pixel values, which produces incorrect results as the three channels are highly correlated with each other. Colour edge detection can be classified into two techniques such as monochromatic-based techniques and vector-valued techniques [1]. There are several types of colour edge detection methods and one of them is reduced ordering (R-ordering). Trahanias and Venetsanopoulos [2] proposed a vector order statistics edge detector based on reduced ordering. minimum vector dispersion based on reduced ordering is shown to be good but it cannot produce an estimate of edge direction. The entropy index is also used in edge detection and Economou [3] introduced an edge detection algorithm based on local, non-parametric estimation of image density, which regards edges as a set of points separating two coherent regions. The method locates and estimates the value of the density minima at region boundaries as a measure of edge strength.

This problem of incorrect filtering due to processing of threecolour channels separately is solved by treating the pixel values as hypercomplex vectors in colour image convolution filters, where the three-colour channels are processed simultaneously. It was first reported that red, green, and blue (RGB) colour components can be processed by encoding the three channel RGB components on the three imaginary parts of a quaternion simultaneously [4]. The hypercomplex convolution operator also has the property of geometrical operations such as stretching, reflecting, rotating, and multiple shears on colour image pixel values. The first example of a colour edge detector was investigated in 1998 [4], in which colour image pixels are represented as pure quaternions and they are multiplied by left and right quaternion coefficients in a convolution. The left and right coefficients are used because of the non-commutative nature of quaternion multiplication, and it allows geometrical operations such as rotation. However, further developments (Evans et al., [5], Sangwine and Ell [6], Sangwine [7], Sangwine et al. [8, 9], Ell and Sangwine [10], Pei and Cheng [11], Jin and Li [12], and Said et al. [13] did not achieve a significant success due to the lack of a well-defined mathematical framework. Only a few researchers worked in this field such as Denis et al. [14] who developed a geometrical idea for spectral content filtering of colour images; moreover, Shi and Funt developed the first texture segmentation using quaternions [15] but they did not develop any new idea. In 2007, Ell devised a key mathematical idea (in Section 2.2), which proved to be unique in the development of linear colour vector filters. He also suggested the use of homogeneous [16] coordinates to express colour image pixels (in Section 2.2.3). The idea for applying the theory of LQSs was advised for such filters in [17]. Although vector image filtering has many applications to date but it did not had a sound theoretical framework based on ad hoc approaches.

In this paper, we have shown that the geometric operations such as rotation on samples or pixels can be expressed in a canonic form and this is a significant step toward the development of linear colour vector image filters. In the formalism of the canonic form, it requires at most four quaternion coefficients. Generally, any linear vector filter can be reduced to a sum of four convolutions, each based on quaternion coefficients or a series or parallel combination of such filters. Many geometrical operations in three-dimensional (3D) can be encoded as a LQ monomial map such as rotations, reflections and simple dilations (see Kuipers [18, p. 345]. The coefficients are $4 \times 4$ matrices, or members of the general linear group of order four, in the formalism of matrices and groups. The proposed filter is formed by combining four LQS (hypercomplex) filters in parallel, reducing the result to the canonic form.

The main contributions of our paper are as follows:

i. First time an ad hoc LQ filter is proposed. 
ii. This paper represented how multi-directional edges can be detected using a single mask.

iii. It presents a theory for multiple geometrical operations combined in a single mask.

iv. The proposed filter is linear as convolution with a quaternionvalued mask is a linear operation and it is also carried out in homogeneous coordinates.

v. The theory reported here is a significant attempt for development of linear colour vector image filters.

vi. It provides a large set of possibilities for design of such filters based on the LQSs framework.

This paper is structured as follows. In Section 1, there are brief descriptions of quaternions and LQSs, in Section 1 we explain the working of the proposed filter, in Section 2, we show the experimental results, produced by applying our proposed filter on colour images in Section 3, and finally in Section 4 we conclude our comments on this paper.

\section{Preliminaries}

\subsection{Quaternion}

The quaternions are a division algebra, which means they have a multiplicative inverse property, and without this property numerical algorithms such as those used in image and signal processing, could not be handled. This work uses the hypercomplex numbers of Hamilton [19], known as the quaternion four-tuple $(w, x, y, z)$ represented in hypercomplex form as

$$
q=w+x i+y j+z k
$$

where, $w, x, y, z \in \mathbb{R}$. The hypercomplex operators obey the operation

$$
i j k=i^{2}+j^{2}+k^{2}=-1
$$

A quaternion, $q \in \mathbb{H}$, can be divided into scalar and vector parts such as

$$
q=s+v
$$

where $s=w=S[q]$ is the scalar part and $v=x i+y j+z k=V[q]$ is the vector part. A conjugation is represented by an overbar which negates the vector part $\bar{q}=s-v$. Generally, three-space vectors are represented as quaternions with a zero scalar part, called pure quaternions, denoted by $V[\mathbb{H}]$.

\subsection{Linear QSs}

In the formalism of LQS, the canonic form consists of at most four quaternion coefficients. In the formalism of matrices and groups, the coefficients are $4 \times 4$ matrices or members of the general linear group of order 4 . In this paper, we have combined four LQS filters in parallel, which reduce to the canonic or minimal filter in homogeneous coordinates.

Real linear functions can be represented as the monomial form

$$
f(x)=m x+c,
$$

where $x, m, c \in \mathbb{R}$.

Linear combinations, direct sums or compositions, of such functions would always be reduced to the same form. However, quaternion linear functions would also have a multinomial form [20]

$$
f(q)=\sum_{p=1}^{P} m_{p} q n_{p}
$$

where all factors are quaternion valued, $q, m_{p}, n_{p} \in \mathbb{H}$.
A quaternion is associated with a four-tuple of reals such as $(a, b, c, d), a_{1}+b_{i}+c_{j}+d_{k}$ and the canonic LQ function would be associated with four-tuple of quaternions as

$$
\{A ; B ; C ; D\}, \quad A q+B q_{i}+C q_{j}+D q_{k}
$$

The four-tuple of quaternions are used to represent the canonic form.

Generally, LQ functions may contain an arbitrary number of terms but they can always be reduced to at most four terms. This reduced function, called the quaternion canonical form of the function, can be completely specified with a four-tuple of quaternions. It is well known that vector rotation and dilation (stretching and compression) and reflections in 3D can be encoded as a LQ monomial map [21]. Rotations of 3D vectors $\boldsymbol{p}$ are encoded with a unit quaternion $q$ in the well known LQ equation [22]

$$
R_{q}[\boldsymbol{p}]=q \boldsymbol{p} \bar{q}
$$

The axis of the rotation corresponds to the eigenaxis of the quaternion $q$ and the angle of rotation is twice the eigenangle of $q$. The composition of two rotations $R_{q}[]$ and $R_{r}[]$ is as follows:

$$
R_{r}[R q[\boldsymbol{p}]]=r(q \boldsymbol{p} \bar{q}) \bar{r}=(q r) \boldsymbol{p}(\bar{q} r)
$$

Finally, the inverse rotation, $R_{q}^{-1}[]$, of $R_{q}[]$ is as follows:

$$
R^{-1} q[\boldsymbol{p}]=\bar{q} \boldsymbol{p} q
$$

2.2.1 LQS convolution operation: In this section, we explain how linear filters may be constructed using LQSs as point operators (the operators that operate on individual samples of the signal or image being processed). We are assuming here linear time-invariant filters (signal processing) or linear shift-invariant filters (image processing) characterised by a finite impulse response or a finite coefficients' mask. Making these assumptions, a filter can be represented as a convolution (we assume here a 1D filter for simplicity and generalisation to two dimensions is simple)

$$
y(n)=\sum_{m=1}^{M} h(m) x(n-m)=h \times x
$$

where $h(m)$ is the impulse response of the filter.

In the case of vector signals and quaternion coefficients, we need four products in the convolution

$$
\begin{aligned}
y(n)= & \sum_{m=1}^{M}((A(m) x(n-m)+B(m) x(n-m) i \\
& +C(m) x(n-m) j+D(m) x(n-m) k)) \\
= & A \times x+B \times x i+C \times x j+D \times x k
\end{aligned}
$$

where $A(m)$ is the $m$ th sample of $A$ and $A$ is a finite quaternionvalued function with $N$ quaternion samples and similarly for $B, C$ and $D$. In this way, we construct a filter from the sum of four quaternion-valued convolutions, three of which are multiplied on the right by the constant values $i, j$ and $k$.

An alternative and higher-level view of the filter is to consider it as the convolution of the signal $x$ with a finite LQ function $F=A+B i+C j+D k$ like this

$$
y=F \times x
$$

Now, at each sample point in $F$, we have a LQ function which implements some geometrical operations and we regard the filter as the convolution of these geometrical operations with the vector signal. 


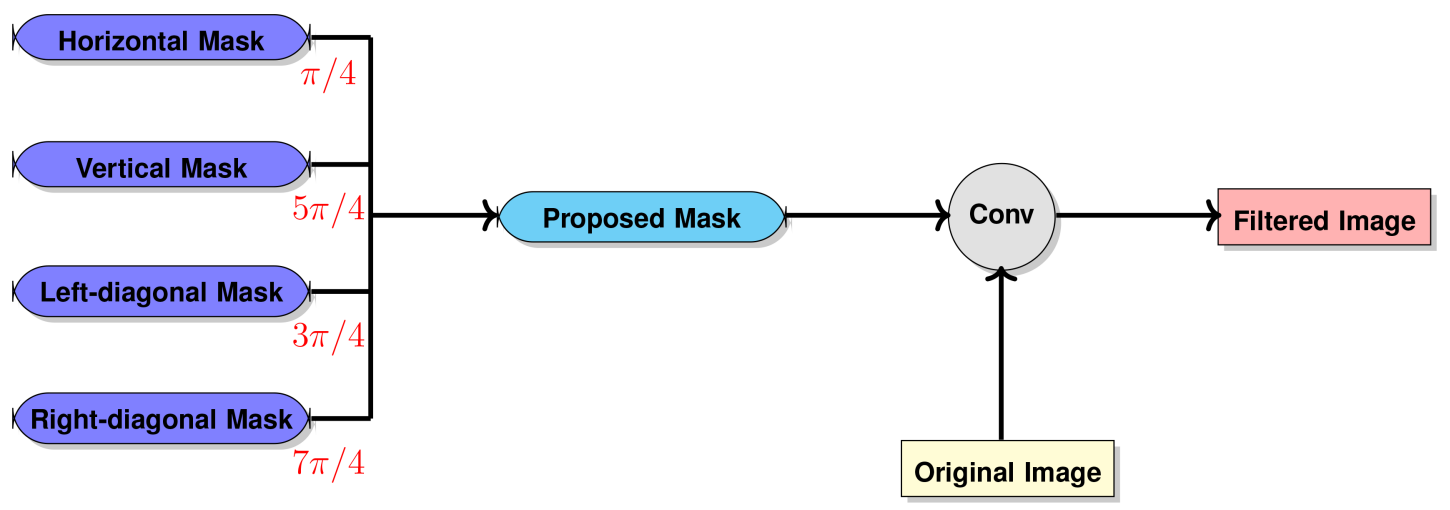

Fig. 1 Parallel combination of filters

2.2.2 Parallel and series combinations: The linear sum of two such functions is calculated by the component-wise addition suppose

$$
\begin{aligned}
& f_{1}(q)=A_{1} q+B_{1} q i+C_{1} q j+D_{1} q k \\
& f_{2}(q)=A_{2} q+B_{2} q i+C_{2} q j+D_{2} q k
\end{aligned}
$$

then

$$
f_{1}(q)+f_{2}(q)=A_{3} q+B_{3} q i+C_{3} q j+D_{3} q k
$$

where

$$
\begin{aligned}
& A_{3}=A_{1}+A_{2} \\
& B_{3}=B_{1}+B_{2} \\
& C_{3}=C_{1}+C_{2} \\
& D_{3}=D_{1}+D_{2}
\end{aligned}
$$

The composition of two linear functions, $f_{2}\left(f_{1}(q)\right)$, is as follows:

$$
f_{2}\left(f_{1}(q)\right)=A_{3} q+B_{3} q i+C_{3} q j+D_{3} q k
$$

where

$$
\begin{aligned}
& A_{3}=A_{2} A_{1}-B_{2} B_{1}-C_{2} C_{1}-D_{2} D_{1} \\
& B_{3}=A_{2} B_{1}+B_{2} A_{1}-C_{2} D_{1}+D_{2} C_{1} \\
& C_{3}=A_{2} C_{1}+B_{2} D_{1}+C_{2} A_{1}-D_{2} B_{1} \\
& D_{3}=A_{2} D_{1}-B_{2} C_{1}+C_{2} B_{1}+D_{2} A_{1}
\end{aligned}
$$

This composition rule shows that it has the same structure as the standard quaternion multiplication. The composition is not commutative: $f_{2}\left(f_{1}(q)\right) \neq f_{1}\left(f_{2}(q)\right)$.

2.2.3 Homogeneous coordinates: A point is represented by coordinate values on 3D axes $(x, y, z)$ (distance in three mutually perpendicular directions from an origin) in Euclidean coordinates of 3D space, while a fourth value is added, known as the weight $(w)$ in homogeneous coordinates. Therefore, a point is expressed by four coordinates $\left(w, x^{\prime}, y^{\prime}, z^{\prime}\right)$ such as $x=x^{\prime} / w, y=y^{\prime} / w$ and $z=z^{\prime} / w$. For converting from Euclidean to homogeneous coordinates, $w=1$.

It is assumed that the images to be processed have samples in a 3D colour space. The theory presented here, based on LQSs, requires that the pixel values have been coded into homogeneous coordinates. The encoding requires a weight to be assigned to each pixel and this is done by arbitrarily assigning a weight of 1 to each pixel value. When representing pixel values in quaternion form, the weight is represented by the scalar part of the quaternion. For example, to encode an RGB pixel value with components $(r, g, b)$ into a quaternion representation in homogeneous coordinates, they are simply encoded as

$$
1+r i+g j+b k
$$

The decoding process requires that all the pixel values be normalised to the same weight. Therefore, taking the same example, we may have a pixel with value

$$
w+r^{\prime} i+g^{\prime} j+b^{\prime} k .
$$

Now, we have to divide it by $w$

$$
1+\frac{r}{w} i+\frac{g}{w} j+\frac{b}{w} k
$$

for getting the pixel value

$$
\left(\frac{r}{w} i+\frac{g}{w} j+\frac{b}{w} k\right)
$$

Translations, affine transformations and projections are linear in homogeneous coordinates, which means these operations could be combined with other operations by matrix multiplication or composition of LQSs. However, the scalar part plays a unique role in homogeneous coordinates.

\section{Working operation of the proposed filter}

Monomial mapping of rotation creates LQS coefficient $L$ from left and right quaternion monomials $R$ and $R^{*}$ coefficients [23]. Similarly, $L^{*}$ from right and left quaternion monomials $R^{*}$ and $R$ coefficients. The LQS operator $L[] L^{*}$ defines a rotation in threespace about the axis $\mu$ through an angle $\pi / 2$ as was discovered by Hamilton [24]. Conjugation of the values $L$ and $L^{*}$ reverses the sense of the rotation. Here, the axis $\mu$ of rotation is the 'grey line' in RGB space which extends from black to white: pixels with values $r=g=b$ fall on this line. The upper and lower rows of the first LQS filter's mask rotate pixel values within RGB space to $+\pi / 4$ and $-\pi / 4$ of their original positions and total is $\pi / 2$, in a plane normal to the grey line, as shown in Fig. 1. In terms of hue, saturation and intensity coordinates, this rotation is a hue shift. In image areas where the upper and lower rows of the masks cover pixels of very similar colour, the rotations of the upper and lower rows add to produce an achromatic pixel value on or near to the grey line. In contrast, when the pixel values under the upper and lower rows differ in hue, as happens at edges in the image, they do not cancel in this chromatic sense. Pixel values with similar hues but different intensities, also cancel chromatically and the filter is insensitive to intensity edges, as shown in Fig. 2.

Similarly, the vertical LQS filter detects vertical edges, which rotate pixel values within RGB space to $+5 \pi / 4$ and $-5 \pi / 4$ of their original positions and total is $5 \pi / 2$, in a plane normal to the grey line. In the same way, the left diagonal LQS filter detects left diagonal edges, which rotate pixel values within RGB space to $+3 \pi / 4$ and $-3 \pi / 4$ of their original positions and total is $3 \pi / 2$, in a plane normal to the grey line. Similarly, the right-diagonal LQS filter detects right-diagonal edges, which rotate pixel values within 

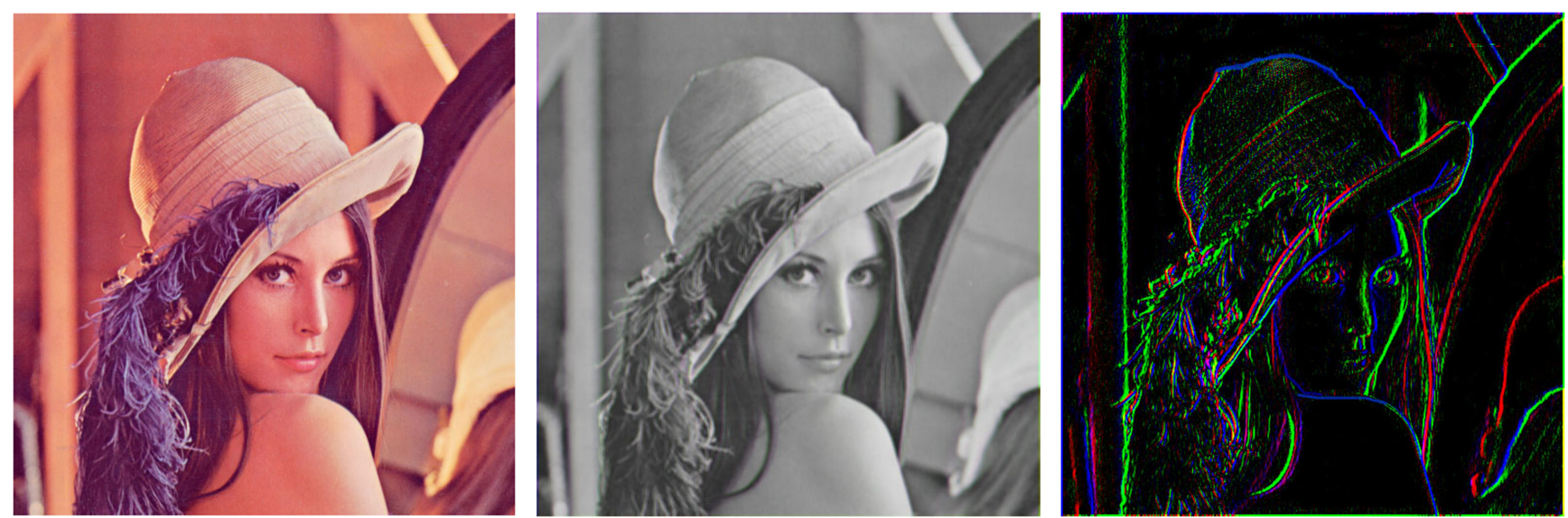

Fig. 2 Original Lena image (left), filtered Lena image by the proposed filter (middle) and filtered chrominance Lena image by the proposed filter (right)
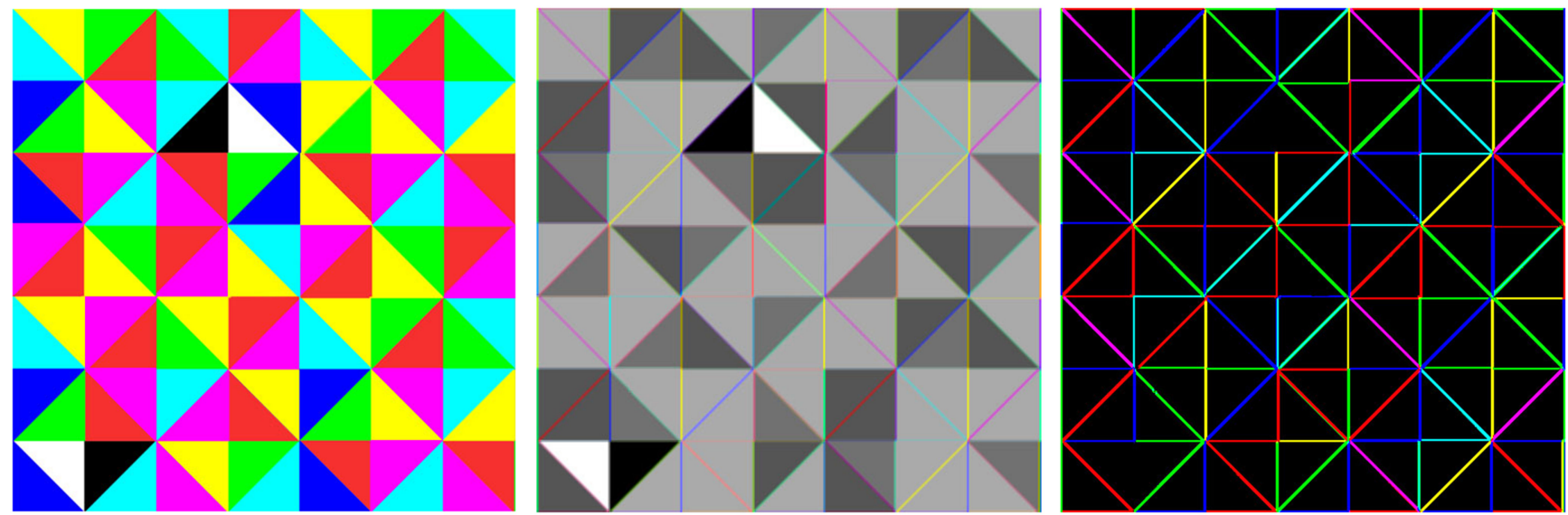

Fig. 3 Original test image (left), filtered test image by the proposed filter (middle) and filtered chrominance test image by the proposed filter (right)

RGB space to $+7 \pi / 4$ and $-7 \pi / 4$ of their original positions and total is $7 \pi / 2$ rotation, in a plane normal to the grey line.

The colour generated by the filter at an edge between two colours is mid-way between the colours in the hue sense. Reversing the sense of the filter by interchanging $L$ and $L^{*}$ in the masks changes the directions, in which the two colours are rotated. Thus, with one sense of the filter, an edge between red and green will yield yellow in the filtered image, while with the other sense, blue (the opponent colour to yellow) will result. The edge colour is defined by the sudden change of hue/colour and the detected edge colour totally depends on it. The more detail, which colour edge should be appeared/detected, is clearly observed from the filtered test image, as shown in Fig. 3. The four LQS masks are combined in parallel to make a single LQS mask. This carries out a single LQS convolution equivalent to four quaternion convolutions. Each multiplication of a mask coefficient with a pixel requires four quaternion multiplications, each of which requires 16 real multiplications and 12 additions. Thus, our LQS filter produces a result without having to combine results from four separate filters and lengthy procedural algorithms. In the classical approach, we have to find each edge direction with individual mask and each convolution is carried out with a separate mask. Then the detected edges are combined using the gradient formula as

$$
\begin{gathered}
|M|=\sqrt{M_{\mathrm{h}}^{2}+M_{\mathrm{v}}^{2}} \\
\theta_{M}=\tan ^{-1}\left(\frac{M_{\mathrm{v}}}{M_{\mathrm{h}}}\right) .
\end{gathered}
$$

where $\theta_{M}$ is the angle of detected edges, $|M|$ is the gradient and $M_{\mathrm{h}}$ and $M_{\mathrm{v}}$ are the horizontal and vertical edges, respectively.

Similarly, with a quaternion filter, we can only find each directional edge with a separate quaternion convolution mask.
However, in our proposed filter, we skip all these procedural algorithm steps and use a single LQ (LQS) convolution mask.

The reason for the four angles chosen is to avoid their cancellation effect when they are combined in parallel. The detected edges appear in primary and secondary colours because of the different rotation angles. Each angle of rotation in a quaternion convolution mask was $45^{\circ}$ rotation in each of four homogeneous coordinates. Masks are reduced/combined into a single mask in parallel. Parallel combination is a weighted sum of pixel values. These chosen angles of rotation are selected for each convolution mask in each four different homogeneous coordinates. That is why they rotate independently of each other and do not cancel out their filtering effects.

A key point is to use homogeneous coordinates because operations on colour images are then linear. A filter consisting of a single quaternion mask has only four degrees of freedom, whereas an LQS mask consists of four quaternions, and therefore has 16 degrees of freedom. Hence, more flexibility is possible in the design of linear colour image filters. Since, in our paper, we use LQSs containing four quaternion-valued LQS convolutions filters, combined in parallel to form a final filter, it is capable of detecting the edges in four different directions such as horizontal, vertical and left- and right-diagonal edges simultaneously.

The proposed filter can be generalised to handle more directions of edges if we increase the size of the convolution mask. For example, a $3 \times 3$ mask can only detect horizontal, vertical, $45^{\circ}$ directional edges of both left and right diagonals. If we use a $5 \times 5$ mask, then it can also detect (45/2)23.5 edges in both left and right directions. For a $7 \times 7$ mask, it can also detect $(23.5 / 2) 11.75^{\circ}$ edges in both left and right directions. As we increase the size of convolution mask, the number of directions of detected edges increases with more enhancing effects but it also increases blurring. by 


\begin{tabular}{|c|c|c|}
\hline $\boldsymbol{L}_{h}^{*}$ & $\boldsymbol{L}_{h}^{*}$ & $\boldsymbol{L}_{h}^{*}$ \\
\hline $\mathbf{0}$ & $\mathbf{0}$ & $\mathbf{0}$ \\
\hline $\boldsymbol{L}_{h}$ & $\boldsymbol{L}_{h}$ & $\boldsymbol{L}_{h}$ \\
\hline
\end{tabular}

\begin{tabular}{|c|c|c|}
\hline $\boldsymbol{L}_{v}$ & $\mathbf{0}$ & $\boldsymbol{L}_{v}^{*}$ \\
\hline $\boldsymbol{L}_{v}$ & $\mathbf{0}$ & $\boldsymbol{L}_{v}^{*}$ \\
\hline $\boldsymbol{L}_{v}$ & $\mathbf{0}$ & $\boldsymbol{L}_{v}^{*}$ \\
\hline
\end{tabular}

\begin{tabular}{|c|c|c|}
\hline $\boldsymbol{L}_{d 1}$ & $\mathbf{0}$ & $\boldsymbol{L}_{d 1}^{*}$ \\
\hline $\mathbf{0}$ & $\mathbf{0}$ & $\mathbf{0}$ \\
\hline $\boldsymbol{L}_{d 1}$ & $\mathbf{0}$ & $\boldsymbol{L}_{d 1}^{*}$ \\
\hline
\end{tabular}

\begin{tabular}{|c|c|c|}
\hline $\boldsymbol{L}_{d 2}$ & $\mathbf{0}$ & $\boldsymbol{L}_{d 2}^{*}$ \\
\hline $\mathbf{0}$ & $\mathbf{0}$ & $\mathbf{0}$ \\
\hline $\boldsymbol{L}_{d 2}$ & $\mathbf{0}$ & $\boldsymbol{L}_{d 2}^{*}$ \\
\hline
\end{tabular}

Fig. 4 Horizontal LQS mask (first), vertical LQS mask (second), left diagonal LQS mask (third) and right-diagonal LQS mask (fourth) of the proposed filter (from left to right)

$$
\begin{aligned}
L & =f\left(R, R^{*}\right) \\
L^{*} & =f\left(R^{*}, R\right)
\end{aligned}
$$

where

$$
R=S \exp ^{\mu \theta}=S(\cos \theta+\mu \sin \theta)
$$

with

$$
\begin{aligned}
S & =1 / \sqrt{x} \\
S_{\mathrm{h}} & =S_{\mathrm{v}}=6 \\
S_{d} i & =S_{d} 2=4 \\
\mu & =(i+j+k) / \sqrt{3} \\
\theta_{\mathrm{h}} & =\pi / 4 \\
\theta_{\mathrm{v}} & =5 \pi / 4 \\
\theta_{d 1} & =3 \pi / 4 \\
\theta_{d 2} & =7 \pi / 4
\end{aligned}
$$

$R^{*}$ is the conjugate of $R, \mu$ is the unit (pure) quaternion and $f(\cdot)$ is the monomial function as defined in (see Kuipers [18, p. 345]: the subscripts $h, v, d 1$ and $d 2$ indicate horizontal, vertical, left and right diagonals. While $S$ is a scale factor to account for the addition of $x$ pixel values, $S_{\mathrm{h}}$ and $S_{\mathrm{v}}$ for horizontal and vertical masks and $S_{d_{1}}$ and $S_{d_{2}}$ for left and right-diagonal masks.

\section{Experimental results}

The experimental results showed that our filter is capable of detecting colour edges in multiple directions such as horizontal, vertical and diagonal edges with only one LQS convolution mask. The proposed filter consists of four LQS filters, which are combined in parallel and the convolution is carried out in homogeneous coordinates.

It is seen in the filtered Lena image, there are two coloured (green and reddish) edges on the hat. Areas of smoothly varying colour become achromatic in the filtered image while the sudden change of colour produce coloured edges, as shown in Figs. 2 and 3 . Thresholding to separate the coloured edges from the achromatic areas is simple and easy using a chromatic threshold. Note that, where the original image had a step change from black to white or vice versa, the filtered image has a grey or achromatic line. The coloured edges in the filtered image have a colour dependent on the colour difference across the edge. As we know that hue/colour depends on the angle of rotation, which causes for the detection of all coloured edges (primary and secondary coloured edges) in the quaternion rotation convolution operation such as $\pi / 4$ rotation in first coordinate, $\pi / 4$ rotation in second coordinate (equivalent to $3 \pi / 4$ ), $\pi / 4$ rotation in third coordinate (equivalent to $5 \pi / 4$ ) and $\pi / 4$ rotation in fourth coordinate (equivalent to $7 \pi / 4$ ).

We apply orthogonal plane decomposition of a quaternion on the filtered Lena and Test images using quaternion MATLAB toolbox [25] to get the chrominance component of the filtered images. Chrominance component is perpendicular decomposition part of the filtered image when axis of rotation of grey line is chosen, as shown in Figs. 2 and 3.
Denis et al. [14] introduced a saturation/gradient colour filter, which is independent from the path (left-wise or right-wise) applied to convolve the filter with the image while Sangwine's filter cannot [4]. The gradient colour filter is used to filter the horizontal, vertical and both diagonal directions. Then, the maximum of these values of saturation at each pixel of the image is chosen to design the final colour gradient filter by maximum distance. The quaternionic filtering operation is linear but the total process is not linear as the 'maximum' operator interferes. Denis's method handles shadows very well but it compares saturation only and cannot distinguish them as efficiently as real contours. This is why the differences in luminance between colours are not detected. Since thresholding is done on the colour gradient to thin detected edges but shadows contours are erased from the edge map. Fig. 5 shows the results of Denis's experiment: left image is the colour gradient, middle image is the log colour gradient (colour gradient image is amplified to amplify the edges detected by the colour gradient filter) and finally the edge map images (it is produced by thresholding the colour gradient). We compare our approach with Denis et al. [14] approach in Fig. 5 and it is shown that our filter is better in performance than Denis's filter.

\section{Conclusion}

We have reported a new type of holistic colour image filter, which can detect horizontal, vertical, left- and right-diagonal edges simultaneously. We have combined four quaternion-based convolution masks into one mask in parallel and the proposed filter detects coloured edges without combination of results from four separate edge detectors. The new filter possesses 16 degrees of freedom of operation in detecting four different directions of edges of a colour image with only a single LQS mask. A very important and significant feature of the proposed filter is that it is a linear filter because convolution with a quaternion-valued mask is a linear operation and the operation is carried out in homogeneous coordinates. The filter theory presented in this paper is a significant step in linear colour vector image filters, which opens a large set of possibilities of the design of such filters based on the LQSs framework. The LQSs operating on homogeneous coordinate representations of the samples or pixels contributes a very useful and general linear framework. These kinds of filters have great usage in the design of efficient aero-dynamic shapes and bruteforce procedural search algorithms of high-resolution images. The proposed filter can be employed on colour or other multi-spectral images and it is the first example of a filter based on LQ convolution (LQS). The theoretical concept and understanding the structuring ideas of systems reduction in a single system using LQS discover a research area in colour vector image processing, especially for linear vector filters [26]. 

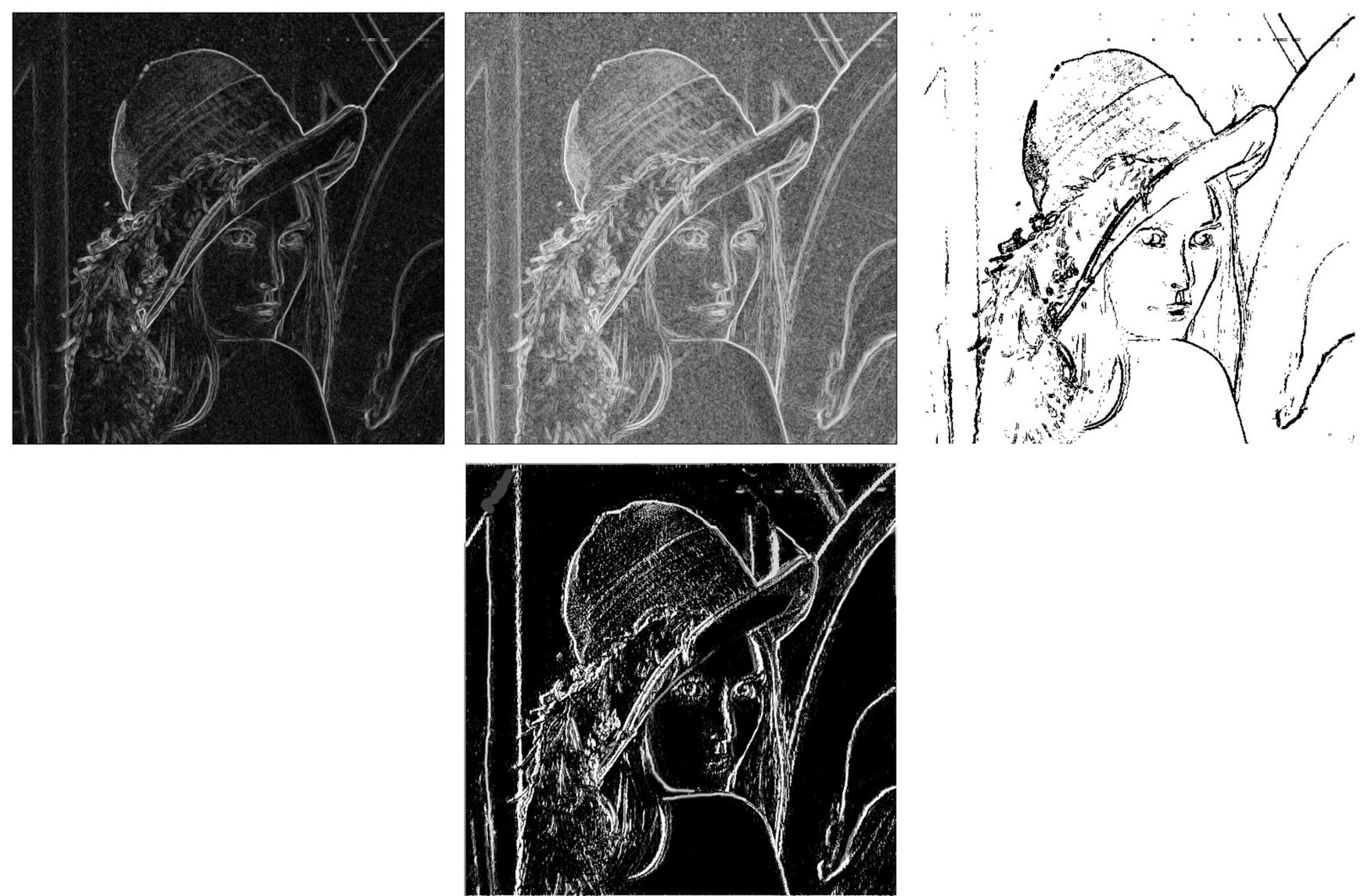

Fig. 5 Top row: results of analysing the Lena image using the method of Denis [14]; from left to right, by colour gradient, log colour gradient (done by amplifying the edges detected by the colour gradient filter) and edge map (done by thresholding on the edges detected by the colour gradient filter). Second row, centre: edge map from our proposed filter

\section{References}

[1] Koschan, A., Abidi, M. 'Detection and classification of edges in color images', IEEE Signal Process. Mag. 2005, 220, (1), pp. 1528-1530. Available at http://dx.doi.org/10.1109/MSP.2005.1407716

[2] Trahanias, P.E., Venetsanopoulos, A.N.: 'Color edge detection using vector order statistics', IEEE Trans. Image Process., 1993, 20, (2), pp. 259-264. Available at http://dx.doi.org/10.1109/83.217230

[3] Economou, G.: 'Detecting edges using density value', Electron. Lett., 2004, 400, (24), pp. 1528-1530. Available at http://dx.doi.org/10.1049/el:20046741

[4] Sangwine, S.J.: 'Colour image edge detector based on quaternion convolution', Electron. Lett., 1998, 340, (10), pp. 969-971. Available at http://dx.doi.org/10.1049/el:19980697

[5] Evans, C.J., Sangwine, S.J., Ell, T.A.: 'Hypercomplex color-sensitive smoothing filters'. Proc. 2000 Int. Conf. Image Processing (ICIP), Vancouver, British Columbia, Canada, September 2000, 1, pp. 541-544. Available at http://dx.doi.org/10.1109/ICIP.2000.901015

[6] Sangwine, S.J., Ell, T.A.: 'Colour in i i image processing', Electron. Commun. Eng. J., 2000, 12, (5), pp. 211-219

[7] Sangwine, S.J.: 'Colour image filters based on hypercomplex convolution'. IEE Proc., Vis., Image Signal Process., 2000, 1470, (2), pp. 89-93

[8] Sangwine, S.J., Gatsheni, B.N., Ell, T.A. 'Vector amplifications for color dependent image filtering'. Proc. 2003 Int. Conf. Image Processing (ICIP), Barcelona, Spain, September 2003, pp. 129-132. Available at http:// dx.doi.org/10.1109/ICIP.2003.1246633

[9] Sangwine, S.J., Ell, T.A., Gatsheni, B.N.: 'Colour-dependent linear vector image filtering'. Proc. 12th European Signal Processing Conf. (EUSIPCO), Vienna, Austria, September 2004, pp. 585-588

[10] Ell, T.A., Sangwine, S.J.: 'Projective-space colour filters using quaternion algebra'. 2008 16th European Signal Processing Conf., 2008, pp. 25-29

[11] Pei, S.C., Cheng, C.M.: 'Color image processing by using binary quaternionmoment-preserving thresholding technique', IEEE Trans. Image Process., 1999, 80, (5), pp. 614-628. Available at http://dx.doi.org/10.1109/83.760310

[12] Jin, L., Li, D.: 'An efficient color-impulse detector and its application to color images', IEEE Signal Process. Lett., 2007, 140, (6), pp. 397-400. Available at http://dx.doi.org/10.1109/LSP.2006.887840
[13] Said, S., Bihan, N.L., Sangwine, S.J.: 'Fast complexified quaternion Fourier transform', IEEE Trans. Signal Process., 2008, 560, (4), pp. 1522-1531. Available at http://dx.doi.org/10.1109/TSP.2007.910477

[14] Denis, P., Carré, P., Fernandez-Maloigne, C.: 'Spatial and spectral quaternionic approaches for colour images', Comput. Vis. Image Underst. 2007, 1070, (1-2), pp. 74-87, ISSN 1077-3142. Available at https://doi.org/ 10.1016/j.cviu.2006.11.019

[15] Shi, L., Funt, B.: 'Quaternion color texture segmentation'. Comput. Vis. Image Underst., 2007, 107, (1-2), pp. 88-96

[16] Meserve, B.E.: 'Fundamental concepts of geometry' (Dover, New York, 1983), Corrected reprint of 1959 edition

[17] Sangwine, S.J.: 'Perspectives on color image processing by linear vector methods using projective geometric transformations, advances in imaging and electron physics', 175 (Academic Press, Cambridge, MA, 2013), pp. 283 307, chapter 6. Available at http://dx.doi.org/10.1016/ B978-0-12-407670-9.00006-8

[18] Kuipers, J.B.: 'Quaternions and rotation sequences' (Princeton University Press, Princeton, NJ, 1999)

[19] Hamilton, W.R. 'Lectures on quaternions' (Hodges and Smith, Dublin, 1853). Available at https://archive.org/details/cu31924062612043

[20] Ell, T.A.: 'On systems of linear quaternion functions'. Preprint available a http://www.arxiv.org/abs/math/0702084v1, February 2007

[21] Ell, T.A.: 'Hypercomplex color affine filters'. IEEE Int. Conf. Image Processing (ICIP) 2007, San Antonio, TX, USA, September 2007. Available at http://dx.doi.org/10.1109/ICIP.2007.4379812

[22] Altmann, S.L.: 'Rotations, quaternions, and double groups' (Oxford University Press, Oxford, 1986)

[23] Ell, T.A., Sangwine, S.J.: 'Linear quaternion systems toolbox for MATLAB', 2007. Available at http://lqstfm.sourceforge.net/

[24] Hamilton, W.R.: 'Elements of quaternions' (Longmans, Green and Co., London, 1866)

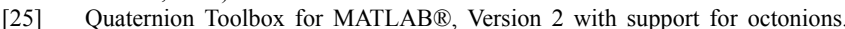
Online, 2013. Available at http://qtfm.sourceforge.net/

[26] Ward, J.P.: 'Quaternions and Cayley numbers: algebra and applications, mathematics and its applications', vol. 175 (Springer, Dordrecht, 1997). Available at http://dx.doi.org/10.1007/978-94-011-5768-1 